\title{
APPLICATION OF HYPERFINE INTERACTIONS TO SURFACE INVESTIGATIONS
}

\author{
M. Budzyński, J. Sarzyński and R. Wasiewicz \\ Institute of Physics, M. Curie-Skłodowska University \\ Pl. M. Curie-Skłodowskiej 1, 20-031 Lublin, Poland
}

\begin{abstract}
In this article the principles of measurements of hyperfine interaction parameters at a given depth in a sample are presented. The advantages and the limitations of the Mössbauer spectroscopy, perturbed angular correlations and nuclear magnetic resonance are described.
\end{abstract}

PACS numbers: $63.35 .-p, 76.80 .+y, 76.60 .-k$

\section{Introduction}

The surface is defined as a boundary between a solid state and a vacuum. The break of symmetry, characteristic of any crystallographic structure, occurs here. Certainly, it is accompanied by a change of physical values, which depends on the local surrounding of nuclear probes. It is, for example, a change of the energy of atomic nuclei, caused by the interaction of their electromagnetic moments with internal magnetic fields and electric field gradients, i.e. hyperfine interaction [1]. The most often applied method are: the Mössbauer spectroscopy (MS), time-differential perturbed angular correlation (TDPAC) and nuclear magnetic resonance (NMR). Hyperfine interactions have a local character. It means that they depend only on the nearest surrounding of nuclear probes and on their configuration. During the deposition of atoms or molecules at the solid state surface, a change of the greater part of parameters occurs in several monolayers. It causes the interest in a study of monoatomic layers. From physical point of view, the deposited layer is a structure with a diminished dimension; two-dimensional on a boundary between solid state and vacuum. The interaction between deposited atoms (nuclear probes in our case) occurs simultaneously through both media. It differs from the interaction between unbounded atoms and between atoms admixtured in a bulk. 


\section{Measurements of hyperfine interaction at the surface and near-surface layer}

\subsection{The Mössbauer spectroscopy in situ}

In the study on surface (being understood at large), the observation of hyperfine interaction dependence on the depth of nuclear probes location is of importance. The most reliable results are obtained by their direct location at a given depth by growing from molecular beam. This method assures a well-defined and almost ideal surface. But it makes high technological demands. The necessary condition is to produce and to keep a vacuum better than $10^{-8} \mathrm{~Pa}$ during the sample preparation and during the measurement.

The rule of this method may be presented by an example of a Mössbauer isotope ${ }^{57} \mathrm{Fe}$. Because of a small value of cross-sections for scattering of $\gamma$-radiation $(14.4 \mathrm{keV})$ in $\mathrm{Fe}$, in photoelectric $\left(\sigma_{\mathrm{ph}}\right)$ and Compton $\left(\sigma_{\mathrm{C}}\right)$ phenomena, the dominant role is played by the resonant absorption $\left(\sigma_{0}\right)$. Since $\sigma /\left(\sigma_{\mathrm{ph}}+\sigma_{\mathrm{C}}\right) \approx 300$, the absorption of the Mössbauer radiation is resonant, in practice, and occurs only in the ${ }^{5}$ Te layer (Fig. 1).
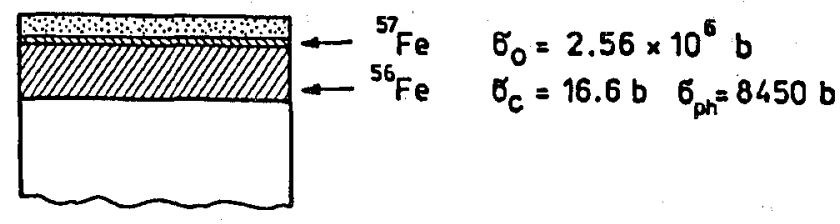

Fig. 1. Sample profile with the ${ }^{57} \mathrm{Fe}$ layer placed at the appropriate depth.

The sensitivity of this method can be increased by a registration of electrons instead of $\gamma$-radiation, due to large coefficient of internal conversion $\alpha_{\text {tot }}=$ $N_{\mathrm{e}} / N_{\gamma} \approx 8.2$. For registration of conversion electrons, the amount of Fe atoms equal to $10^{13}-10^{14}$ per $\mathrm{cm}^{2}$ is sufficient. It stands $0.1-0.01$ of monolayer. However, the measurement needs about one day. After such time it is difficult to speak about "pure" surface even in a vacuum $10^{-10} \mathrm{~Pa}$.

At present, measurements of the Mössbauer spectra are performed mainly for ${ }^{57} \mathrm{Fe},{ }^{119} \mathrm{Sn}$, and ${ }^{151} \mathrm{Eu}$.

\subsection{Depth selective conversion electron Mössbauer spectroscopy (DCEMS)}

Another possibility to measure the dependence of hyperfine interaction parameters at different depths of nuclear probe is the Mössbauer spectroscopy of nuclear radiation, emitted by the deexcitation of $14.4 \mathrm{keV}$ state in ${ }^{57} \mathrm{Fe}$. It consists of some groups of internal conversion electrons $(K, L, M)$, Auger electrons $(K-L L, L-M M), \gamma$-radiation and X-rays $(K, L)$ of various energy and intensity. Because of different values of absorption of electromagnetic and corpuscular radiation, the layer at various depths can be measured. The Mössbauer spectroscopy of $\gamma$-radiation gives information about internal fields in a bulk sample, while registration of X-rays allows to study layers of $10-15 \mu \mathrm{m}$ thick. 
From measurements of electrons of internal conversion, new quantitative information can be obtained about near-surface layer of homogenic samples of bulk type. After all, about $90 \%$ of all electrons in a standard iron sample came from a layer of thickness about $100 \mathrm{~nm}$. The depth of electron penetration range can be estimated from the well-known formula

$$
I=I_{0} \mathrm{e}^{-\mu x} \text {, }
$$

where $\mu$ is an absorption coefficient of an electron beam in medium.

In the case of electrons with kinetic energy $5-25 \mathrm{keV}$ the following empirical relation is useful [2]:

$$
\mu=4.43 \times 10^{2} \frac{d}{E^{3 / 2}},
$$

where $d$ is the density of medium $\left[\mathrm{g} / \mathrm{cm}^{3}\right]$ and $E$ is the initial energy of electrons [keV]. Thus $m$ is expressed in $\mathrm{nm}^{-1}$.

A direct application of formula (1) is impossible because of statistical character of electron scattering in solid state. The angular distribution of the converted electrons and Auger electrons generated in the sample is isotropic. Moreover, electrons emitted from nucleus, which are located at a given depth, reach the surface along incidental way. The simulations of electron trajectories with different energies in Fe matrix were performed in [3]. In spite of this, in nonresonant scattering of $\gamma$-radiation (by atomic shell), electrons with anisotropic angular distribution are emitted. In photoelectric phenomena, for small value of $\gamma$-quanta, the angular distribution of electrons is described by a function $f(\theta) \approx \sin ^{2} \theta$, where $\theta$ is an angle between $\gamma$-ray direction and momentum vector of photoelectrons. In the Compton scattering, a well-known angular dependence occurs.

The theory and experimental technique of DCEMS studies were developed mainly by Liljequist and co-workers [3] for ${ }^{57} \mathrm{Fe}$. It assumes a relationship between the final energy of electrons and the path passed by them in a sample. It is necessary to determine the energy of electrons of the Mössbauer spectrum with high accuracy. It is not easy, remembering that the energetic selection for a high-transmission spectrometer is difficult. The probability of registration of electrons belonging to the definite group (for example $K$ ) with the kinetic energy $\mathrm{E}_{k} \pm \mathrm{d} E_{k}$, emitted from a depth $x$ to the solid angle on the surface $G \pm \mathrm{d} G$ is determined by the function $T^{k}\left(x, G, E_{k}\right) \mathrm{d} G \mathrm{~d} E_{k}$. These functions for various $x$, for fixed $E_{k}$ and $G$, are called weight functions. Knowledge of weight functions for the medium under study is an example of application of a given method, especially when we want to know the exact value of the depth $x$ from which our electrons come. The justification requires a preparation of some samples, in which the resonant isotope is placed at given depth. Sometimes only a knowledge of changes of the hyperfine interaction parameters is sufficient.

\subsection{Scattering of the Mössbauer radiation at glancing angles (GA-DCEMS)}

Scattering of the Mössbauer radiation which is incident at the surface at small angles (near the critical angle) is worthy of notice. The wavelength of the Mössbauer radiation is comparable with a lattice constant. Thus, a crystal lattice 
may be treated as a three-dimensional diffraction grating. Under the scattering of electromagnetic radiation a coherent multiplication of waves scattered by individual atoms takes place. As a result of it, the diffracted wave becomes coherent with the incident wave. Interference of the incident wave and of the reflected one leads to the total internal reflection. It happens closer and closer to the surface as the angle decreases. This method allows to perform measurements for any sample with a properly smooth surface and it requires an intensive collimated beam of the Mössbauer radiation. Figure 2 shows a schematic experimental equipment.

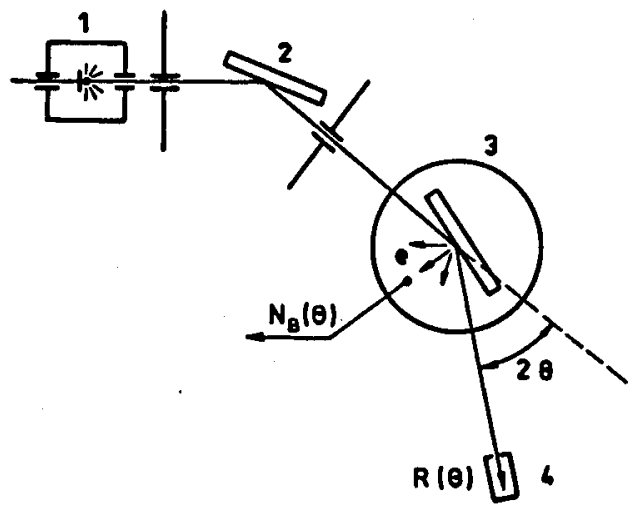

Fig. 2. Schematic experimental equipment for the scattering of the Mössbauer radiation at small angles. 1 - source fixed on vibration pivot, 2 - monochromator-collimator $\mathrm{Ge}(220), 3$ - proportional counter for detection of electron with a sample inside it, 4 - detector for reflected beam of $\gamma$-radiation.

The angular dependence of a reflected beam $R(\theta)$ and of an incident beam $T(\theta)$ is given by the relations known from wave optics [4]:

$$
\begin{aligned}
& R(\theta)=\frac{\left[\sin \theta-\left(\chi+\sin ^{2} \theta\right)^{1 / 2}\right]^{2}}{\left[\sin \theta+\left(\chi^{2} \theta\right)^{1 / 2}\right]^{2}}, \\
& T(\theta, \chi)=\frac{4 \sin ^{2} \theta \exp [-q(\theta) x]}{\left[\sin \theta+\left(\chi+\sin ^{2} \theta\right)^{1 / 2}\right]^{2}},
\end{aligned}
$$

where

$$
q(\theta)=\frac{4 \pi}{\lambda} \operatorname{Im}\left(\chi+\sin ^{2} \theta\right)^{1 / 2} .
$$

In these formulae $T(\theta, x)$ means the intensity of the wave field in a sample at the depth $x, \theta$ is an incident angle, $\chi=\chi+i \chi_{\mathrm{i}}$ is a complex coefficient of the medium polarization, $q(\theta)$ is the attenuation coefficient and $\lambda$ is the wavelength of incident radiation.

Because the depth penetration of $\gamma$-radiation is rather long, this method is not applicable to the assignment of a task. However, one should exercise a possibility given by the registration of low-energy electrons which reach the surface. One can normalize, for a given angle, the amount of electrons of definite energy which 
reach the surface to the amount of electrons obtained under the perpendicular incidence of $\gamma$-radiation

$$
K(\theta, E)=\frac{N_{\theta}(\theta, E)}{N_{0}(\pi / 2, E)}=\frac{1}{\sin \theta} \int_{0}^{\alpha} T(\theta, x) P(x) \mathrm{d} x,
$$

where $P(x)$ is the transmission function in request. Of course,

$$
\int P(x) \mathrm{d} x=1 \text {. }
$$

The angular dependence of the emission of electrons reaching the surface from deeper layers of the sample (with lower energies) will be diminished in comparison to the emission of electrons from near-surface layers (high energies). This method is not elaborated properly. A widespread application of it requires some improvements and new developments, mainly as far as numerical description of experimental data is concerned.

At present, the most valuable results are obtained by a deposition of nuclear probes on the surface or at a definite depth. This manner is commonly carried on not only in the Mössbauer spectroscopy but also for the measurements of time differential perturbed angular correlations (TDPAC) and of nuclear magnetic resonance (NMR).

Because the hyperfine magnetic fields in two-dimensional structures as the subject matter were presented during the previous conferences devoted to surface physics, we will focus our attention on the multilayer structures.

Recently, investigations are concentrated on the propagation of the ferromagnetic ordering in the structures with an artificially constructed periodicity. This idea is applied to the creation of artificial fields and to modification of magnetic properties of initial elements and of thin films of alloys.

Multilayer structures of iron and rare-earth elements are most often studied [5]. We paid much attention to $\mathrm{Fe}-\mathrm{Al}$ system. The investigation started with the multilayered structures $\mathrm{Fe}-\mathrm{Al}$. The multilayered films were prepared by sequential deposition of two elements. Al layers of constant thickness $32 \AA$ were placed between Fe layers of stable thickness varying from sample to sample. Each of the samples was covered by $\mathrm{Al}$ coat $300 \AA$ thick to protect it against oxidation. The deposition was performed in ultra high vacuum conditions, with the mica plates as the substrata.

The Mössbauer measurements reveal that ferromagnetic ordering occurs when the Fe layers thickness is equal to $16 \AA$, at least. It results from the ratio of amplitudes of the Zeeman sextuplet that ferromagnetic domains are initially arranged in the plane of the sample. At the Fe layer thickness equal to $32 \AA$ the process of random orientation of domains occurs.

$$
\begin{aligned}
& \text { 2.4. Application of time differential } \\
& \text { perturbed angular correlations } \gamma-\gamma(T D P A C)
\end{aligned}
$$

It appears that for the investigation of the surface by TDPAC method the sufficient concentration of nuclear probes ${ }^{111} \mathrm{In}$ is of the order of $10^{-4}$ monolayer (ML). It means two orders of magnitude lower than requirements of CEMS. It 
is clear because during the time of measurements comparable to the half-life $\left[T_{1 / 2}\left({ }^{111} \mathrm{In}\right)=2.8\right.$ days $]$ almost each nuclear probe gives a signal which can be registered.

The ${ }^{111}$ In isotope is widely applied to measurements of quadrupole splitting. The electric field gradient at a surface is a result of the broken symmetry of the solid at the surface and therefore it depends on the surface orientation. This is confirmed by the results of Klas et al. [6].

Very interesting question is the temperature dependence of the electric field gradient at the surface and its behaviour in the bulk. In many bulk materials of noncubic structure this temperature variation exhibits a $T^{3 / 2}$ dependence. It is commonly accepted that the mean-square displacement of nuclear probes and their immediate neighbours are responsible for this effect. At the crystal surface the two-dimensional structure gives rise to an anisotropic vibrational behaviour resulting in anisotropic displacements of the surface atoms. That should influence the temperature dependence of the surface field gradient. Such a reasoning is confirmed by the experimental results. They indicate a linear temperature dependence

$$
V_{z z}(T)=V_{z z}(0)(1-B T),
$$

where a constant $B$ is of the order of $10^{-4} K^{-1}$.

A similar temperature dependence for the electric field gradient has also been noticed for some of bulk materials, for example $\mathrm{NbSe}_{2}$, which has a quasi-two-dimensional layer structure. However, some deviations from the linear dependence of the electric field gradient at a surface have been observed for ${ }^{111} \mathrm{In}$ on $\mathrm{Cu}$ (100) [6].

TDPAC method allows the observation of nuclear probes behaviour after their deposition on the real surface, for example [6].

\subsection{Application of NMR method to the surface study}

Application of NMR method to the surface study is difficult. The detection of population of the splitted sublevels for resonance frequency requires high amount of nuclear probes. It is known that the amount of attainable probes may be smaller than amount of atoms in the monolayer (on the average about $10^{15}$ atoms $/ \mathrm{cm}^{2}$ ). This is too little for the application at traditional solutions.

In the first measurements the crumbled samples with small size of the grains were used. It secured the suitably high ratio of atoms placed at the surface to these placed in the bulk. It was assumed that the detected signal originated from the developed surface.

Some improvements allowed direct measurements for the single crystal. The results obtained for ${ }^{23} \mathrm{Na}$ deposited at the tungsten surface may serve as an example [7]. Parameters of the electric field gradient tensor depend on the surface

$$
\begin{array}{ll}
\mathrm{W}(100): & V_{z z}=-25 \times 10^{15} \mathrm{~V} / \mathrm{cm}^{2} \perp \text { to the surface, } \\
\mathrm{W}(110): & V_{z z}=-54 \times 10^{15} \mathrm{~V} / \mathrm{cm}^{2} \quad \perp \text { to the surface, } \\
\mathrm{W}(112): & V_{z z}=40 \times 10^{15} \mathrm{~V} / \mathrm{cm}^{2} \quad \| \text { to the surface. }
\end{array}
$$




\section{Summary and perspectives}

The method in situ is the simplest and the most reliable method of measurements of hyperfine interaction parameters. It allows a study of properties of superlayers and multilayers. Application of this method requires a subtle technique of preparation of monoatomic layers with a coating close to one. There exist possibilities to produce and to study magnetic structures with reduced dimension, i.e. ultrathin film monolayers (2D) and linear chain (1D). Surprisingly, early studies [8] reported that $\mathrm{Fe}, \mathrm{Co}$, and $\mathrm{Ni}$ surface layers were magnetically "dead". Now it is well-recognized that environments with few nearest neighbours and hence weaker interatomic hybridization are conductive to enhanced magnetization [9].

At the same time, a great development of theoretical methods is observed. They are able to predict, basing on the first principles, the greater part of observed effects $[10,11]$. It seems that the most effective method to calculate the electronic structure is the method of full potential linearized augmented plane wave (FLAPW). It is suitable for thin layers as well for bulk structures. Knowledge of electronic structure allows to determine magnetic moments of individual atoms, energies of magnetic anisotropy as well as parameters describing the hyperfine fields.

In the future, perhaps ultrathin layers will be applied as an information carrier, because of magnetization which is perpendicular to a layer plane. It occurs frequently in ultrathin epitaxial Fe layers [12], as a result of the break of symmetry and surface anisotropy.

\section{References}

[1] Hyperfine Interactions of Radioactive Nuclei, Ed. J. Christiansen, Springer-Verlag, Berlin 1983.

[2] R.A. Krakowski, R.B. Miller, Nucl. Instrum. Methods 100, 93 (1972).

[3] D. Liljequist, M. Ismail, Phys. Rev. B 31, 4131 (1985).

[4] A.J. Chumakov, G.V. Smirnov, Zh. Eksp. Teor. Fiz. 89, 1810 (1985).

[5] L.T. Baczewski, M. Piecuch, J. Durand, G. Marchal, P. Delcroix, Phys. Rev. B 40, 11237 (1989).

[6] T. Klas, R. Fink, G. Krausch, R. Platzer, J. Voigt, R. Wesche, G. Schatz, Surf. Sci. 216, 270 (1989).

[7] U. Memmert, D. Fick, Surf. Sci. 203, 276 (1988).

[8] L.M. Lieberman, J. Clinton, D.M. Edwards, J. Mathon, Phys. Rev. Letl. 25, 232 (1970).

[9] A.J. Freeman, R. Wu, J. Magn. Magn. Mater. 100, 497 (1991).

[10] A.J. Freeman, C.L. Fu, J. Appl. Phys. 61, 3356 (1987).

[11] A.J. Freeman, R. Wu, J. Magn. Magn. Maler. 104-107, 1 (1992).

[12] D. Pescia, Europhys. News 21, 103 (1990). 